\title{
Periodontal Screening and Recording
}

National Cancer Institute

\section{Source}

National Cancer Institute. Periodontal Screening and Recording. NCI Thesaurus. Code C114641.

A system for assessing the health of the gums as based on the depth of the gingival sulci. The periodontal screening and recording (PSR) index divides the mouth into 6 segments (sextants) and the greatest probe depth in each sextant of the mouth is determined and recorded. 